\title{
Annotation \\ The Effect of Small Samples on Crown-Size Interrelationships
}

\author{
Stanley M. Garn, Patricia E. Cole, and Kenneth E. Guire \\ Center for Human Growth and Development and Department of Anthropology, University of \\ Michigan, Ann Arbor, Michigan 48109 USA
}

J Dent Res 56(12): 1474, December 1977.

In a recent paper Lavelle $(J$ Dent Res 56:541, 1977) reported both differing magnitudes and patterns of mesiodistal-buccolingual crown-size correlations in 3 human races and in samples of Pan, Pongo, and Gorilla, using 20 males in each case. Others have called attention to differences in crown-size correlations $(r)$ and variances $\left(\sigma^{2}\right)$ from similarly small archeological samples (Suarez, AmJ Phys Anthrop 41:411, 1977).

While the sampling distributions of a single $r$ are well known, such results are difficult to interpret in the context of correlated values of $r$ derived from a single sample.

Accordingly, we have taken a computer simulation approach, using successive independent samples from a larger population and calculating mesiodistal-buccolingual correlations $(r)$ for each tooth. The data base consisted of 109 males and 99 females, described by MOYERS ET AL (Standards of Human Occlusal Development, 1976). A computer-based program was used to draw five completely random subsamples of 20 individuals of each sex (Fox and Guire, Documentation for Midas, 1976, p 82). In question was the extent to which mesiodistal-buccolingual correlations could vary in magnitude or in pattern from sample to sample.

For the five random subsamples for each sex, restricted to individuals with complete and measurable dentitions, differences in mesiodistal-buccolingual correlations for each of 14 teeth ranged from as little as 0.12 to as much as 0.73 . The largest range of MD$\mathrm{BL} r$ 's for a single tooth extended from -0.45 to \pm 0.28 in girls. In addition, there were wide subsample variations in the relative values of $r$ for anterior and posterior teeth, as shown here for males (Figure).

In all, $74 \%$ (31) of the mesiodistal-buccolingual correlations given by Lavelle for three races of man fell within the range established for males within a single base population. Moreover, $62 \%$ (26) of the MD.BL correlations given for Pan, Pongo, and Gorilla fell within these same subsample ranges. This

Received for publication Augus 10, 1977

Accepted for publication: September $27,1977$.

This study was supported. in part, by Ciran DI:-03610 irom the National lumlitise of Health.

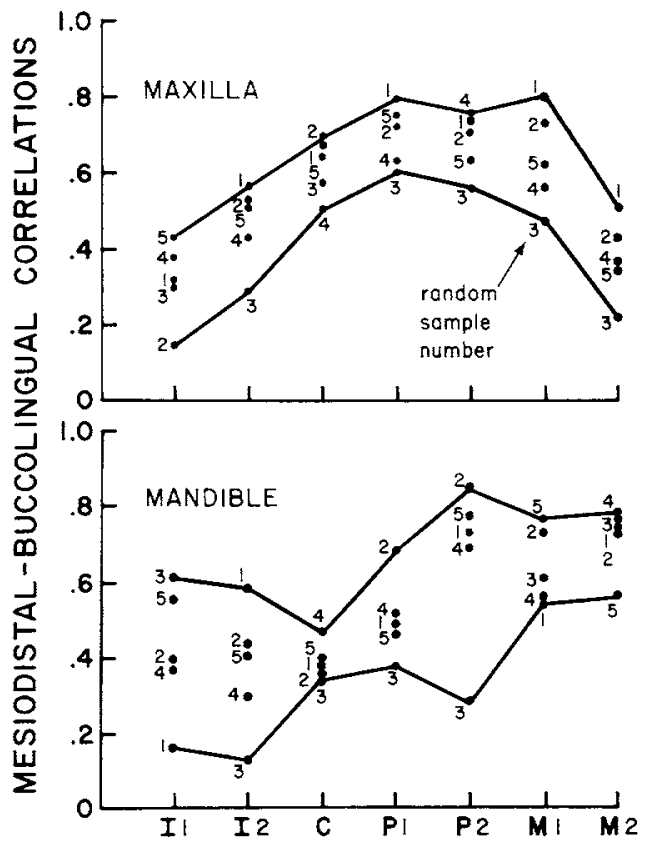

Range of mosiodisat-buecolingut correlation (slamn by con. nected lines) and subsample values of $r$ (shown by subample litm. bery from five successive, independent random subsample of 20 malcs cash, computer-derived from a larger data bane.

study, which simulates naturally occurring small samples, indicates the analytical hazards in generalizing from limited odontological material, whether recent or fossil. Similar problems exist even with conventional means and percentiles, as we have shown in another simulation study (GARN ET AL, Ecol Food Nutr $6: 153,1977)$. 